\section{$\underset{\substack{\text { hommes } \\ \text { \& migrations }}}{ }$}

\section{Hommes \& migrations}

Revue française de référence sur les dynamiques

migratoires

\section{$1321 \mid 2018$}

Les mots de l'exil dans l'Europe du XIXe siècle

\title{
Figures de l'exil dans les chansons et poésies communardes
}

\author{
Laure Godineau
}

\section{(2) OpenEdition \\ Journals}

Édition électronique

URL : http://journals.openedition.org/hommesmigrations/4300

DOI : 10.4000/hommesmigrations.4300

ISSN : 2262-3353

Éditeur

Musée national de l'histoire de l'immigration

Édition imprimée

Date de publication : 1 avril 2018

Pagination : 85-91

ISBN : 978-2-919040-41-4

ISSN : $1142-852 X$

Référence électronique

Laure Godineau, «Figures de l'exil dans les chansons et poésies communardes », Hommes \& migrations [En ligne], 1321 | 2018, mis en ligne le 01 avril 2020, consulté le 06 janvier 2021. URL: http://journals.openedition.org/hommesmigrations/4300 ; DOI : https://doi.org/10.4000/ hommesmigrations.4300 


\title{
FIGURES DE L'EXIL \\ DANS LES CHANSONS ET POÉSIES COMMUNARDES
}

Par LAURE GODINEAU, maîtresse de conférences en histoire contemporaine, université Paris-13

\author{
Si les chansons et les poèmes ont chanté la Commune de Paris \\ en 1871 et la geste des communards, ce chant ne s'est pas tari \\ avec la fin de l'insurrection. Au contraire, il a suivi le chemin des \\ anciens insurgés dispersés aux quatre coins du monde. Ce \\ corpus de textes inspirés rend compte ainsi du partage de la \\ souffrance, de l'absence et de l'éloignement de la terre natale \\ entre exilés et déportés. À travers leurs mots, l'exilé se charge \\ d'une nouvelle et paradoxale dimension : tout en restant \\ l'absent, en vie parmi les morts, il est aussi le dernier témoin \\ de l'histoire.
}

Dernière révolution parisienne du XIX ${ }^{e}$ siècle, mouvement insurrectionnel et populaire réprimé dans le sang, devenu très vite mythique, la Commune de Paris de 1871 a donné lieu à une profusion d'écrits ${ }^{1}$. Dès le début de l'insurrection, les littératures anticommunarde et pro-communarde se sont opposées, prolongeant sur le papier les combats armés. À la suite de la répression, dans les années 1870, cette production s'est maintenue, avec bien sûr une différence quantitative nette, puisque la parole des insurgés ou de leurs partisans, en France, s'est en partie tarie avec les arrestations et les poursuites, les procès et les condamnations qui ont couru jusqu'au milieu de la décennie.

Au total, plus de 40000 prisonniers ont été jugés, et plus de 10000 condamnations ont été prononcées contradictoirement par les tribunaux militaires. La Nouvelle-Calédonie a été établie comme lieu de déportation en 1872 et un peu plus de 3800 condamnés y ont été envoyés, condamnés soit à la déportation simple à l'île des Pins, soit à la déportation dans une enceinte fortifiée à la presqu'île Ducos. S'y sont ajoutés plus de 200 condamnés aux travaux forcés, transportés au bagne de l'île $\mathrm{Nou}^{2}$. Il y a eu un peu plus de 3300 condamnations par contumace et on estime que le nombre d'exilés dans les différents paysrefuges se situe autour de 5 à 6000 , en GrandeBretagne, en Suisse, en Belgique, ou dans d'autres pays d'Europe ou d'autres continents comme l'Amérique ${ }^{3}$.

Cet exil au sens large a duré jusqu'aux amnisties partielle et plénière votées successivement en mars 1879 et juillet 1880. En exil, un certain nombre de militants ont pris la plume pour revenir sur l'insurrection et sa défaite. À côté des ouvrages et des 
écrits divers sur l'expérience de la Commune, la "Semaine sanglante » mais aussi la répression qui a suivi ont été des thèmes privilégiés par les acteurs de 1871 et leurs sympathisants, et ce jusqu'à l'amnistie. Après l'exil, le retour a été l'occasion de publier en France des œuvres restées inédites jusque-là. Cette production laisse une place non négligeable au genre particulier des chansons et poésies, objet d'analyse de cet article. Il s'agit donc de s'arrêter ici sur l'utilisation poétique et non politique ou administrative, juridique, des mots de l'exil, en privilégiant par ailleurs non pas l'angle de l'accueil, mais celui des acteurs.

\section{Une poésie à géographie variable}

L'importance de la chanson pendant la guerre de 1870 et la Commune a été mise en lumière depuis longtemps déjà, notamment par Robert Brécy en 1991. Dans son ouvrage qui est également une anthologie, ce dernier aborde la chanson pendant les années d'exil et dans les années postérieures, en France et dans les pays-refuges. Plus récemment, Philippe Darriulat est revenu sur le sujet en traitant de la mémoire immédiate de 1871 dans la chanson populaire ${ }^{5}$. Par ailleurs, au début des années 1950, Eugène Schulkind consacra une thèse sur la littérature de la Commune et notamment les poèmes ${ }^{6}$. Son étude, qui porte surtout sur la littérature communarde comme arme de combat, permet néanmoins de revenir en partie sur les traces écrites des déportés et des transportés en Nouvelle-Calédonie.

Ces travaux soulignent bien que différents moments se succèdent dans la chronologie des chansons et poésies de l'absence. En France, la Commission de censure sur les œuvres dramatiques et les chansons destinées aux théâtres et aux concerts, qui avait été supprimée par le Gouvernement provisoire, est rétablie en 1871. Elle doit examiner le contenu des chansons proposées par les éditeurs et les directeurs de salles. Après la Commune, la plupart de celles qui évoquent de près ou de loin l'insurrection sont refusées. Les chansons de café-concert ont donc peu de chance de mentionner la Commune et l'exil jusqu'en 1875, voire après. De même, très peu de chansons de colportage ont trait à l'insurrection. Cependant, même si beaucoup de pièces en vers ne passent pas la censure, le changement est sensible avec les progrès des républicains dans les urnes à partir de 1876 et surtout un peu avant l'amnistie. D'autres, évoquant la Commune, en nombre très limité, circulent par le colportage, notamment en province. Certaines d'entre elles, qui se trouvent dans les cartons des archives de la Préfecture de police, ont courtcircuité le dépôt légal et la censure, d'autres sont chantées en privé. En 1871-1872, par ailleurs, des chansons et des poèmes sont rédigés en prison par des acteurs de l'insurrection, par Louise Michel par exemple, qui les joint parfois à ses lettres, ou par des personnages moins connus ou anonymes, mais ne sont bien sûr pas imprimés à cette période.

En dehors de la France, en revanche, des pièces de vers sont écrites dès le début de l'exil par des réfugiés et éditées à Bruxelles, à Londres ou à Genève. Le plus souvent, ces textes portent sur les événements de la guerre de 1870 et de la Commune. Apparaissent également des poèmes et des chansons sur la Semaine sanglante mais aussi sur la répression judiciaire, l'emprisonnement, les pontons, et bientôt, notamment à partir de 1872, sur la déportation. Avec l'installation dans l'exil, une fois passées les toutes premières années, c'est notamment lors des anniversaires de la Commune que des chansons traditionnelles ou composées spécialement pour la circonstance sont chantées. Elles ne sont pas toutes publiées, mais il en subsiste des traces, des bribes, parfois le texte entier, dans les archives de police ou dans la presse. Certaines d'entre elles, ainsi que 
d'autres poésies rédigées dans l'exil, paraitront postérieurement à l'amnistie, en recueil?

Enfin, des pièces en vers sont composées en NouvelleCalédonie de 1873 à 1880, et éditées dans la plupart des cas dans les années 1880 ou plus tard encore. Henri Brissac, par exemple, journaliste pendant l'insurrection et condamné au bagne de l'île Nou, fait connaître en 1887 douze poèmes dans son ouvrage Quand jétais au bagne, textes du bagne qu'il dit avoir alors appris par cœur pour les conserver ${ }^{8}$. Jean Allemane, condamné également aux travaux forcés, publie pour sa part une plaquette de ses poèmes en 1926, dont certains sont datés de ses années de bagne'. Quant à Louise Michel, elle a écrit d'assez nombreuses poésies de sa déportation, que l'on ne peut, le plus souvent, dater précisément ${ }^{10}$. Enfin, dans les dernières années de Nouvelle-Calédonie, les déportés simples sont parvenus à produire de petites feuilles, dont L'Album de l'̂̀le des Pins, qui contient des pièces de vers, la plupart sentimentales, mais dont quelques-unes sont plus politiques et centrées sur la déportation et la transportation ${ }^{11}$.

Les recueils parus essentiellement dans les années postérieures à 1880, mais contenant des œuvres antérieures, par exemple ceux de Louise Michel, d'Allemane, d'Eugène Pottier, ou d'Eugène Chatelain ${ }^{12}$, ou d'autres pièces éparses, mentionnées pour la plupart par Robert Brécy dans son ouvrage, constituent l'essentiel du corpus de cet article. Ce corpus ne prétend donc pas à l'exhaustivité. Il permet néanmoins de dégager un certain nombre d'analyses sur les représentations topiques de l'exil et de l'exilé dans ces œuvres communardes ou pro-communardes. Même si les conditions de production ne sont pas les mêmes pour tous et aux différents moments, quelles sont alors les figures de l'exil qui apparaissent dans ces textes?

\section{Une souffrance partagée}

Sans surprise, ces pièces de vers reprennent les topoï de l'absence, de l'éloignement et de la souffrance de l'exilée ${ }^{13}$. La souffrance est de manière traditionnelle attribuée à l'éloignement de la patrie, parfois de la famille. La posture n'a rien d'original. Nombreux sont les poèmes qui reprennent ces figures attendues, tel le "Souvenir » d'Eugène Chatelain, daté de 1876 et présent dans le recueil Les Exilées de 1871, paru en 1886 :

"Il pleuvait. Nous étions aux longs soirs de novembre;

L'exil rivait nos pas sur un sol étranger

Tu vins. Je touvris la porte de ma chambre ;

Ta pensée, aussitôt, se mit à voyager.

Mon âme vagabonde, heureuse du voyage,

Avec la tienne alla vers la France et Paris.

Tes souvenirs, les miens, formèrent le bagage...

Qu'il est doux de penser à ceux qu'on a chéris! $[\ldots]^{14} »$

L'exilé fait savoir son amour de la patrie perdue. Dans l'expérience de 1871, le topos est accentué par le patriotisme affiché du communard, qui revendique un combat contre la traitrise et la capitulation lors dela guerre franco-prussienne, alors que la Commune est accusée par le camp anti-communard d'avoir œuvré à la destruction de la France sous les yeux de l'ennemi germanique, d'avoir fait preuve d'un « cosmopolitisme » suspect, voire d'une collusion avec l'ennemi. En outre, la capitale représente à elle seule l'essence de la patrie, et le patriotisme du communard exilé est avant tout parisien ${ }^{15}$.

Un autre élément spécifique de cette expérience communarde se trouve dans le rapprochement qui

7. Sur les détails de cette chronologie, en France et en exil, voir Robert Brécy, "Des lendemains de la Commune à l’amnistie ", op. cit., pp. 111-215. 8. Henri Brissac, Quand j'étais au bagne, Paris, Dervaux, 1887. 9. Jean Allemane, Mes Chansons, Bergerac, La semeuse, 1926, 16 p., cité par Robert Brécy, op. cit., p. 116. 10. Louise Michel, À travers la vie, Poésies, 1894 et À travers la vie et la mort, ⿷匚uvre poétique recueillie et présentée par Daniel Armogathe et Marion Piper, Paris, Maspero, 1982, réédition La Découverte, 2001. 11. Album de l'île des Pins, 1878-1879, voir Robert Brécy, op. cit., p. 168. 12. Eugène Pottier s'exila en Angleterre puis aux États-Unis; Eugène Chatelain se réfugia principalement à Jersey. 13. Sur les références littéraires et artistiques stéréotypées et, de façon générale, sur les lieux communs utilisés par les exilés au xix siècle, plus particulièrement pendant le Second Empire, voir Sylvie Aprile, Le siècle des exilés. Bannis et proscrits de 1789 à la Commune, Paris, CNRS éd., 2010, notamment chapitre 8. 14. Eugène Chatelain, Les Exilées de 1871. Poésies, Fables, Chansons, Paris, Patay, 1886, p. 216 ; la seconde strophe n'étant pas sans évoquer Les Regrets de du Bellay. 15. Voir à ce sujet l'ensemble des travaux de Jacques Rougerie. Voir aussi Robert Tombs, Paris, bivouac des révolutions. La Commune de 1871, Paris, Libertalia, 2014 (1re édition : The Paris Commune 1871, Londres/New York, Longman, 1999). 
est opéré - nous verrons que c'est là une constante - entre les exilés et les déportés. Dans la souffrance de l'éloignement, ceux qui ont été condamnés - et, partant, souvent déportés - et ceux qui ont échappé à la répression et se sont réfugiés à l'étranger partagent le même sort, le terme d'" exil » pouvant alors être utilisé au sens large. Cette mise en avant d'un sort commun se retrouve en particulier dans de nombreuses pièces de vers en faveur de l'amnistie, dans les dernières années de la décennie 1870. Ainsi, un poème de Jules Jouy, qui deviendra un chansonnier du Chat Noir et qui fait ses débuts à la Lice chansonnière, paraît par exemple dans la feuille Le Sans-Culotte, à la fin de l'année 1878. Accompagné d'un dessin d'Alfred Le Petit, il porte le titre «Amnistie ? » et évoque l'attente douloureuse du déporté/exilé :
"Le déporté, sombre vigie,
Sur la falaise, sa prison,
Est triste comme une élégie.
Son ceil morne, sur l'horizon,
Évoque la noire maison
Où sa femme l'attend encore.
Les bruits de l'atelier sonore,
Tout là-bas, bercent son exil,
Oh pardon! quand de ton aurore
L'Océan resplendira-t-i $i^{16}$ ?"

Le dessin d'Alfred Le Petit n'est d'ailleurs pas sans rappeler la figure de l'exilé isolé sur son rocher, telle que l'avait si symboliquement représentée Victor Hugo à Jersey ${ }^{17}$. Poèmes et chansons, dessins sont imprégnés de l'expérience de cette figure tutélaire des républicains de la seconde moitié du XIXe siècle, qui, dans ces années, mène par ailleurs avec vigueur le combat pour l'amnistie totale ${ }^{18}$. Sur son triste rocher calédonien, faisant écho à celui de l'île anglo-normande, le déporté semble être le symbole de l'exilé de 1871, comme Hugo fut celui des années d'Empire.

\section{Mourir en exil}

C'est la mort dans le pays lointain qui est dès lors le signe de cette souffrance et le témoignage de la cruauté de la répression et de l'absence d'amnistie. Cette patrie qu'ils ont défendue, certains ne la reverront jamais. Le combat pour l'amnistie est aussi politique : cette patrie qu'ils défendaient, c'était également la France républicaine et patriote, mais la République mise en place ne reconnaît pas les siens et laisse mourir ses fils loin d'elle. Ainsi, après l'amnistie partielle et le retour des premiers exilés et déportés entre mars et octobre 1879, et dans le combat pour obtenir l'amnistie plénière, une feuille volante est distribuée par les comités d'aide aux amnistiés ${ }^{19}$, avec deux chansons sur les recto et verso, «Le retour du proscrit » et « À ceux qui restent » (Air de la grâce de Dieu $)^{20}$. Dans la première, on lit :

«(...) Salut douce terre de France

L'amnistie nous a tout rendu.

Moins le proscrit que la souffrance

A cloué sur ce sol perdu! (...)»

Utilisée à des fins politiques, l'image de la douleur mortifère de l'éloignement et de l'attente a de nouveau pour conséquence de lier les exilés et les déportés. Commune aux uns et aux autres, elle revient par conséquent à plusieurs reprises dans les poèmes de Nouvelle-Calédonie. Cependant, c'est alors une mort non seulement fort lointaine, mais aussi perdue dans les mers exotiques qui est évoquée. Et ceux que les souffrances de l'exil auront

16. Jules Jouy, "Amnistie? ", in Le Sans Culotte, décembre 1878, cité par Robert Brécy, op.cit., p. 197. 17. Voir les photographies de Charles Hugo prises à Jersey, notamment Victor Hugo sur le rocher des Proscrits, 1853, Musée d'Orsay; Victor Hugo, photographies de l'exil, Paris, Musée d'Orsay-Maison de Victor Hugo, Paris, 1998. 18. Voir Laure Godineau, Retour d'exil. Les anciens communards au début de la Troisième République, thèse de doctorat en histoire, Paris, université Paris-I, 2000, notamment pp. 68-109. Rappelons aussi l'admiration que lui vouait, par exemple, Louise Michel, qu'Hugo célébra dans son poème "Viro major "; ou encore le parcours particulier d'Eugène Chatelain, qui rappelle dans Les Exilées de 1871 que l'impression de ses premières poésies fut arrêtée en décembre 1851 avec le coup d'État, et qui, après 1871, trouva, tout comme Hugo avant lui, exil à Jersey. Nous remercions ici Sylvie Aprile pour ses remarques sur Victor Hugo lors des discussions de la journée d'études du programme AsileuropeXIX " Les mots de l'exil dans I'Europe du XIXe siècle ", Paris, MNHI, 19 janvier 2017 ; voir aussi Sylvie Aprile, Le siècle des exilés, op. cit., et "Victor Hugo dans le rocher des Proscrits ", in " Ressources iconographiques", AsileuropeXIX, https://asileurope.huma-num.fr. 19. Dépendants du Comité central d'aide aux amnistiés, organisé par les radicaux et présidé par Victor Hugo et Louis Blanc. Se crée aussi un comité socialiste concurrent, de moindre influence. Voir Laure Godineau, Retour d'exil..., op. cit., pp. 137-149. 20. Archives de la Préfecture de police (APP), BA 469 
laissés en Nouvelle-Calédonie resteront seuls, à jamais. Personne ne reviendra sur leurs tombes ou dans leurs cimetières. L'amère désolation revient, par exemple, dans les poèmes de Jean Allemane ou de Louise Michel. Dans le "Chant des transportés ", daté de septembre-octobre 1876, Jean Allemane affirme :

\section{"(...) Exténués, on peut les voir dans l'ombre \\ Debout encor, car l'espoir les nourrit. \\ Ils sont tes fils, ô France bien aimée, \\ Entends leur voix, fais cesser leur douleur ; \\ Mais hâte-toi, la houle désolée \\ Roule des morts dans les coraux en fleur ${ }^{21}$. "}

Le thème est également repris dans un poème publié dans l'Album de l'île des Pins, "Ceux qui resteront » :

"(...) Moi, je pense aux morts que tient ce rivage,

Qu'a tués cette île, et qu'en leurs linceuls,

Dans l'affreux désert de ce roc sauvage,

Il faudra laisser, quelque jour, tout seuls ${ }^{22}$. »

Là encore, l'élaboration de la mémoire de la Commune qui se fait pendant ces années donne à cette mort lointaine une tonalité particulière. En effet, en dehors des divisions personnelles et politiques qui ont existé dès 1871 et se maintiennent ensuite, les communards soulignent à maintes reprises qu'ils étaient une force collective et que la répression les toucha tous, quelles que soient les formes qu'elle prit - la fusillade, l'emprisonnement, la déportation, l'exil.

Dès lors, la mort en exil, et plus particulièrement en Nouvelle-Calédonie, est l'expression d'une souffrance, le témoignage d'une cruauté, mais elle est aussi, pour ainsi dire, une forme ultime de répression, non pas seulement parce qu'elle est la mort, mais parce que dans ces conditions, la mort n'est plus collective - comme elle l'a été pendant la Semaine sanglante - et le mort est détaché du souvenir collectif et des lieux de mémoire collective comme le Père-Lachaise. Il restera « tout seul ».

\section{Déportation et exil}

Dans ce collectif de l'éloignement et de la souffrance créé par l'exil au sens large, une échelle de la douleur est donc malgré tout introduite, dans les chansons et poèmes, entre les exilés au sens strict, les réfugiés dans les différents pays étrangers, et les condamnés à la déportation et au bagne ${ }^{23}$.

Cette distinction émane d'abord des déportés et des transportés eux-mêmes, la Nouvelle-Calédonie étant associée pour l'essentiel à l'horrible peine des travaux forcés, au bagne, y compris chez les déportés. Les différences de peines, déportation simple, déportation dans une enceinte fortifiée, travaux forcés, tendent à s'effacer derrière la pire des condamnations. Or, et le thème revient fréquemment dans les pièces de vers, le bagne est pire que la mort. C'est ce qu'écrit, par exemple, Jean Allemane dans « Le Chant des transportés »:

«(...) En s'apaisant, ô comble d'infamie,

Tes flots soumis les mèneront au port.

Ne pouvaient-ils leur arracher la vie?

Le bagne est-il préférable à la mort? (... $)^{24}$.»

$\mathrm{Ou}$ encore Louise Michel dans «Le chant des captifs » :

«(...) Viens en sauveur, léger navire,

Hisser le captif à ton bord!

Ici, dans les fers, il expire ;

Le bagne est pire que la mort (... $)^{25}$.»

Cette distinction est également reprise par certains exilés comme Eugène Pottier ${ }^{26}$ ou par des partisans des communards condamnés, comme le journaliste et poète provençal Clovis Hugues, qui a lui-même connu une peine de quatre ans de prison en 1871 pour sa participation à la Commune de Marseille. Début 1878, il fait paraître une longue poésie en faveur de l'amnistie, «Les Martyrs », dans laquelle il évoque tour à tour les souffrances de la prison, puis de l'exil, et enfin, au point culminant, la 
Nouvelle-Calédonie. La comparaison, juste esquissée ici, est souvent reprise dans les poèmes et chansons, y compris dans les années 1880: la Nouvelle-Calédonie, c'est le bagne, c'est-à-dire l'Enfer de Dante :

«(...) Oui. Mais l'exil n'est rien encore. Ce qui fait Courir un noir frisson dans lêtre stupéfait,

Ce qui fait suer l'agonie,

Ce qui tord les flancs nus des tragiques damnés,

Ce qui fait regretter aux hommes d'être nés,

C'est l'horrible Calédonie ${ }^{27}$ !"

Dès lors, même si léloignement mortifère de la Patrie et des siens recouvre l'exil au sens large, doit-on conclure que les allusions à la Nouvelle-Calédonie convoquées dans ces œuvres
En effet, en dehors des divisions personnelles et politiques qui ont existé dès 1871 et se maintiennent ensuite, les communards

soulignent à maintes

force collective et que la répression les toucha tous, quelles que soient les formes qu'elle prit - la fusillade,

l'emprisonnement, la déportation, l'exil. reprises qu'ils étaient une introduisent une brèche dans une souffrance revendiquée comme collective? Comme d'autres textes, les pièces de vers cherchent en fait sans cesse, à construire un destin commun à toutes les victimes de la répression, quelles qu'elles soient, proscrits, déportés, et fusillés. Ainsi, au-delà du topos de la souffrance de l'absence, et bien qu'introduisant parfois une échelle de la douleur, elles font de l'exil un élément majeur du collectif communard ${ }^{28}$.

L'exilé au sens strict, celui que l'on qualifie aujourd'hui plus aisément de réfugié, celui qui a échappé à la mort, aux prisons et à la déportation a-t-il donc une place spécifique dans ce collectif de la souffrance?

\section{Le « réfugié », un témoin héroïque}

Dans le cadre de la Commune et de sa répression, il peut occuper une place délicate dans la mesure où il est encore vivant alors que nombre de ses compagnons ont été fusillés, et où il ne connaît pas l'enfer de la Nouvelle-Calédonie. Comment lui faire rejoindre ce collectif, en dépassant le recours traditionnel à la douleur de l'absence ? Ce topos apparaît en effet presque insuffisant dans un moment où l'on met en avant la violence et l'ampleur de la Semaine sanglante, mais aussi la cruauté de la déportation et de la transportation.

Si le réfugié n'est pas mort, s'il ne connaît pas le triste sort de ceux qui ont été pris, nombre des chansons et poèmes des années 1870-1880 lui font connaître un sort semblable à celui de ses compagnons, bien au-delà du sort commun dû à la souffrance de l'éloignement. "L'Exil, c'est la prison qui marche », écrit Clovis Hugues dans « Les Martyrs ${ }^{29}$ ». Plus encore, l'exilé n'est pas seulement un condamné par contumace, mais c'est un mort par contumace. Ainsi, dans son célèbre poème "La Commune de Paris » écrit en 1876, pour le cinquième anniversaire de la Commune, à New York, Eugène Pottier, après avoir évoqué successivement les morts de 1871 et l'agonie en Nouvelle-Calédonie, mentionne la mort cruelle qu'est l'exil :

"(...) Parce que tu nous tiens, nous, morts par contumace,

Dispersés dans l'exil, sans pain et sans travail, Et qu'affolant le riche et pelotant la masse

Tu nous montres de loin comme un épouvantail $(\ldots)^{30} . »$

Bien qu'ayant échappé à l'enfer de la répression, l'exilé réfugié rejoint de facto, dans les mots, ceux qui ont agonisé ou agonisent encore.

Certains poèmes cherchent également à mettre l'accent sur cette communion en montrant que l'exilé réfugié était là en mai 1871, au moment de la Semaine sanglante, comme ceux qui ont été fusillés ou ont été pris, et qu'il a été tout aussi héroïque. Alors que les accusations anti-communardes de lâcheté des meneurs fusent dès 1871, et tandis qu'une mémoire doloriste en construction met l'accent sur l'héroïsme de ceux qui sont morts au 
combat $^{31}$, ces textes recourent, pour fonder cette unité, non seulement au topos de la mort due à l'éloignement, à celui de la mort douloureuse du réfugié due à la dispersion, à la pauvreté et à la stigmatisation - morts par contumace -, mais aussi, par un retournement subtil, à la figure du héros. L'exilé réfugié est une figure héroïque, non pas parce qu'il est mort avec les autres, mais parce que, paradoxalement, il est vivant. Son exil est le signe qu'il a eu plus de chance que ses compagnons, et aussi qu'il a pu s'échapper sans doute avec des complicités ; par conséquent qu'il a été aidé par la population parisienne dans laquelle il s'est fondu, qui l'a reconnu comme l'un des siens. Son exil est d'autre part l'expression d'une volonté, car il a choisi un ailleurs, alors que la France réprime ; le subi se transforme en choisi. C'est ce qu'expriment par exemple les poèmes d'Eugène Chatelain dans Les Exilées de 1871, en particulier «Le Proscrit de 1871 » ou «Adieu aux habitants de Jersey». Le refrain du « Proscrit de 1871 » répète :

"Par les balles, couché par terre,

Je me suis relevé, vaincu

Et depuis ce temps, j’ai vécu

Sous le ciel gris de l'Angleterre ${ }^{32}$.»

Puis viennent des strophes où le personnage, «seul vivant, parmi des morts », est reçu, soigné et caché dans une famille parisienne, ce qui lui permet de gagner ensuite le pays-refuge. De même, dans "Adieu aux habitants de Jersey ", le proscrit rappelle qu'il a

\section{«(...) préféré la mer et l'immense horizon}

Aux grilles d'un cachot dans [sa] pauvre patrie ${ }^{33}$. » Quelles furent, dans les faits, les perceptions et les conditions d'accueil faites aux exilés ? Dès la fin de la Semaine sanglante, Jules Favre, ministre de l'Intérieur, avait demandé aux gouvernements européens l'extradition des réfugiés communards les plus connus. Aucun ne fit suite à sa demande. Le gouvernement belge fit bien des déclarations d'intention hostiles aux exilés, mais Victor Hugo, résidant à Bruxelles, protesta en offrant symboliquement l'asile aux communards. Il fut expulsé mais, in fine, devant à la fois l'opposition des libéraux belges et l'exemple anglais, la réglementation concernant les réfugiés fut assouplie. Pour autant, en Belgique, comme en Suisse, en GrandeBretagne ou dans les autres pays-refuges, nombre d'exilés connurent des difficultés matérielles pendant ces longues années, malgré des exemples de réussites individuelles. La très grande majorité revint une fois les amnisties votées. Ce fut aussi le cas des déportés en Nouvelle-Calédonie.

Le retour accentue l'expérience collective, mais les mots des chansons et des poèmes de l'exil contribuent déjà très fortement à créer une communion entre déportés et exilés réfugiés, qui dépasse les figures attendues de l'éloignement. Cette communion est même poussée jusqu'à l'association avec les morts de la Semaine sanglante. Après les fusillades, c'est la proscription qui fait le collectif communard, là où sans doute la Commune a échoué. Elle est l'élément fédérateur, peut-être même plus que la dernière semaine de mai, plus que les morts de 1871 , dans la mesure où, précisément, exilés et déportés sont vivants. C'est aussi dans cette perspective qu'il faut comprendre les vers qui font des exilés et des déportés, là aussi a priori de manière assez attendue, les futurs acteurs du combat; mais non pas d'un nouveau combat, plutôt du même qui se prolonge. Des «soldats d'avenir » écrit Jean Allemane dans " Le Chant des transportés ${ }^{34}$ », qui regardent néanmoins, nous semble-t-il, sans cesse vers 1871 et les années d'exil. Les mots de l'exil concourent ainsi à façonner un destin commun à tous les insurgés, fait de souffrance mais aussi d'héroïsme, et ce quelle que soit la diversité de l'expérience individuelle, en 1871 et dans les années 1870, et même si une échelle de douleur peut parfois être introduite. In fine, les poésies et chansons de l'absence révèlent, à elles seules, à quel point c'est dans ces années essentielles que se mettent en place les éléments spécifiques de la mémoire de l'insurrection. 